\title{
A Avaliação da Competência Social: Versão Portuguesa da Forma para Professores do SSRS
}

\author{
Marina Serra de $\operatorname{Lemos}^{1} \mathrm{e}$ \\ Helena Isabel Meneses \\ Universidade do Porto
}

\begin{abstract}
RESUMO: O trabalho de investigação aqui apresentado teve um duplo objetivo: explorar as dimensões da competência social em crianças portuguesas e adaptar um instrumento de avaliação válido para a investigação e intervenção nesta área e utilizável em contexto escolar. Nesse sentido, o presente estudo examinou a estrutura fatorial e a consistência interna da escala para professores do Social Skills Rating System - nível básico, de Gresham e Elliott (1990) em 342 alunos do $3^{\circ}$ e $6^{\circ}$ anos de escolaridade. Este instrumento destina-se a avaliar a competência social, baseando-se numa perspectiva multidimensional. Os resultados apóiam uma concepção multifacetada da competência social e são semelhantes aos apresentados em estudos prévios.
\end{abstract}

Palavras-chave: dimensões da competência social; avaliação de alunos portugueses; Social Skills Rating System

\section{The Assessment of Social Competence: Portuguese Adaptation of the SSRS (Teacher Form ) to Children}

\begin{abstract}
The present study had two main goals: (a) to explore the dimensionality of social competence in portuguese students, and (b) to adapt an assessment tool of student social behaviors, useful for research and intervention in the school context- the SSRS (Gresham \& Elliott, (1990). To achieve this, we examined the reliability, and the structural configuration of the SSRS' (Teacher form) constructs, in a sample of 342 3rd and 6th grade students, focusing on whether the responses of Portuguese students reflected the posited multidimensional pattern. Results strongly supported a multifaceted view of social skills, and were compatible with previous empirical findings.
\end{abstract}

Key words: social competence dimensions; Social Skills Rating System; portuguese children

A competência social desempenha um papel fundamental no desenvolvimento humano em geral e no funcionamento adaptado na escola, afetando as relações com os professores, a aceitação pelos pares e a realização acadêmica.

A competência social refere-se a um conjunto de comportamentos aprendidos, socialmente aceites. Uma boa competência social permite interações eficazes com os outros e previne relações socialmente inaceitáveis (Gresham \& Elliott, 1984). As crianças capazes de partilhar, iniciar interações positivas, ajudar, pedir ajuda quando precisam, pedir por favor e agradecer, serão bem sucedidas nas suas relações, o que constitui uma das mais importantes tarefas do desenvolvimento. Em contrapartida, déficits na competência social relacionam-se com baixa realização acadêmica e podem levar a problemas de adaptação futura ou mesmo psicopatologia (Coie \& Dodge, 1983; Cowen, Pederson, Babigian, Izzo, \& Trost, 1972; Garmezey, Masten, \& Tellegen, 1984; Parker \& Asher, 1987).

A competência social é um conceito lato, utilizado para descrever o comportamento social, a compreensão e utilização de habilidades sociais e a aceitação social (Haager \& Vaughn, 1995). Refere-se sobretudo às competências de

1 Endereço: R. do Campo Alegre, 1021-1055, 4150, Porto. interação com o mundo social, embora se encontrem na literatura definições que incluem neste conceito competências mais genéricas; estas, geralmente, constituem requisitos básicos para as primeiras. Há mesmo concepções de competência social tão abrangentes que a vêem como uma capacidade individual para coordenar recursos.

A competência social é um construto multidimensional e interativo. Os componentes considerados nas várias definições dependem das perspectivas teóricas (por exemplo, a da aprendizagem social, a construtivista ou a etológica), sobre o funcionamento e o desenvolvimento social. Analisando a diversidade de definições de competência social, Dodge (1985) identificou dois fatores comuns: a receptividade e disposição para responder aos estímulos do ambiente (por exemplo, a atenção, a sensibilidade e a adequação das reações próprias às circunstâncias) e a eficácia social (i.l.: a habilidade de interagir eficazmente no ambiente social, especialmente com os pares, por exemplo através de estratégias de resolução de problemas e habilidades socio-cognitivas). Estes aspectos comuns e outros componentes específicos são vistos como interagindo na produção do comportamento socialmente competente. A competência social não decorre da simples presença dos seus componentes, mas também das interações entre eles. Trata-se portanto de um construto multidimensional, que inclui fatores sociais/interpessoais, cognitivos e emocionais. Por exemplo, um dos modelos mais 
referidos e estudados (Vaughn \& Hogan, 1990) acentua que o comportamento socialmente competente resulta da integração entre quatro componentes: relações com os pares, cognição social, problemas de comportamento e habilidades sociais eficazes.

\section{Formas de Avaliação da Competência Social}

No plano teórico a investigação deve apoiar-se numa definição conceptual de competência social que transcenda a especificidade de situações, tarefas e idade, no sentido de permitir estudar a sua continuidade e mudança em diferentes contextos e ao longo do ciclo vital. No plano operacional, a definição da competência social deve refletir a variação das suas manifestações (nas suas diversas componentes) em função das situações específicas (Oppenheimer, 1989) e da idade do indivíduo (Markus \& Wurf, 1987; Oppenheimer, 1987). Assim, a avaliação da competência social requer múltiplos informantes e diferentes indicadores, adequados às tarefas de desenvolvimento e características de processamento cognitivo típicos de cada idade. Numa revisão da literatura sobre déficits de competência social Hazel e Schumaker (1988) consideram que a utilização de um único instrumento será sempre redutora na identificação deste tipo de déficit. Um só instrumento não poderá fornecer a informação necessária para detectar déficits de habilidades sociais específicas ou para avaliar de forma precisa os resultados de programas de treino de competência social.

Os procedimentos mais freqüentemente utilizados na avaliação da competência social incluem: (a) a sociometria (Moreno, 1934) e as várias medidas dela decorrentes, tais como a nomeação pelos pares, desenvolvida por Coie (Coie, Dodge, \& Copotelli, 1982), a classificação dos pares (Gresham, 1981b) e a avaliação dos pares (por exemplo a técnica de Kaufman, Agard e Semmel, (1985), em que as crianças avaliam várias características do comportamento dos seus pares); (b) a avaliação feita pelos professores que, embora mais freqüentemente utilizada para a avaliação dos problemas de comportamento, também tem sido um recurso importante para a avaliação das habilidades sociais; para além da escala incluída no estudo que a seguir se apresenta, existem algumas escalas válidas como, por exemplo, a Social Behavior Assessment (Stephens, 1978, 1981), ou a escala WalkerMcConnell (Walker \& McConnell, 1988), (c) os registros de observação da ocorrência de comportamentos específicos (por exemplo, dar "feedback" positivo e negativo, aceitar "feedback" negativo, resistir à pressão dos pares, negociar ou resolver problemas) e da qualidade da interação social; (d) a avaliação feita pelos pais tem sido pouco utilizada nesta área (destaque-se a Social Behavior Assessment-Parents que apresenta características satisfatórias); contudo, na área da psicopatologia (Achenbach, 1982; Edelbrock, 1983; Quay, 1983) e da avaliação da adaptação comportamental (Reschley \& Gresham, 1988) há muita investigação que utiliza os pais como informantes; finalmente, (e) as medidas de auto-relato.

\section{O "Sistema de Avaliação da Competência Social”' (SSRS)}

O Social Skills Rating System (SSRS) é um sistema de avaliação, constituído por diversas escalas de registro de comportamento. Este sistema foi desenvolvido por Gresham e Elliott (1990), no sentido de satisfazer os requisitos acima enunciados para a avaliação da competência social e de servir de base para o planejamento de intervenções nesta área. As escalas que o compõem apresentam características distintivas, que as tornam especialmente pertinentes. Trata-se de um sistema que avalia várias facetas específicas da competência social, agrupadas em duas escalas: as habilidades sociais e os problemas de comportamento. Avalia também a competência acadêmica, que tem sido enfatizada na literatura da especialidade, pelo papel que desempenha nos processos de adaptação e desadaptação social. O sistema integra três escalas: uma escala de habilidades sociais, uma de problemas de comportamento e uma de competência acadêmica. Os itens centram-se em comportamentos positivos e em competências pró-sociais, não deixando de incluir uma breve avaliação de comportamentos potencialmente problemáticos e a competência acadêmica. O sistema completo permite uma avaliação coordenada de informantes múltiplos, que pode incluir professores, pais e os próprios alunos, captando assim indicadores de competência social em diferentes contextos e segundo diferentes perspectivas. Os alunos podem ser percebidos de modo diferente em diferentes ambientes: diferentes informantes podem julgar o seu comportamento de forma diferente ou os alunos podem demonstrar diferentes comportamentos sociais em diferentes contextos. Recorde-se que o contexto influencia o tipo e a estrutura das interações sociais. As escalas são ainda diferenciadas em função do nível de desenvolvimento da criança. As formas para professores e pais têm três níveis cada: uma para crianças dos três aos 5 anos, outra para crianças da pré-escola ao $6^{\circ}$ ano de escolaridade e outra do $7^{\circ}$ ao $12^{\circ}$ ano. A forma para o aluno (auto-relato) existe apenas para dois níveis: uma do $3^{\circ}$ ao $6^{\circ}$ ano e outra do $7^{\circ}$ ao $12^{\circ}$. O sistema comporta também uma escala de importância na qual o informante especifica a importância atribuída a cada comportamento, o que garante a validade social dos comportamentos avaliados e facilita a seleção de comportamentos alvo para a intervenção (como por exemplo os comportamentos com baixa freqüência e importância elevada). Por último, inclui ainda uma folha de registro integrado que permite relacionar os resultados da avaliação com o planejamento da intervenção.

O SSRS original tem uma estrutura fatorial pura e os fatores demonstraram boa consistência interna (Clark, Gresham \& Elliott, 1985; Gresham, Elliott, \& Black, 1987a). A validade de construto tem sido avalizada por estudos que mostram correlações moderadas com o rendimento acadêmico, com problemas de comportamento, com observações naturalistas de interações sociais em sala de aula, com a atração física e com o estatuto sociométrico (Elliott, Gresham, Freeman, \& McCloskey, 1988; Gresham, Elliott, \& Black, 1987b). 


\section{Competência Social}

\section{A Competência Social na Escola}

A competência social na escola tem sido alvo de uma atenção especial. A investigação nesta área tem mostrado que a competência social se relaciona fortemente com a realização acadêmica. Os alunos com dificuldades de aprendizagem e os alunos com um rendimento normal apresentam enormes diferenças na competência social. Comparadas com as crianças sem dificuldades, as primeiras são menos aceitas ou mais rejeitadas pelos pares, revelam déficits em vários comportamentos sociais positivos e demonstram níveis mais elevados de comportamento social negativo (Bryan, 1982; Deshler, Schumaker, Warner, Alley, \& Clark, 1980; Gresham, 1981a; Gresham \& Reschly, 1987; Gresham \& cols., 1987a). A investigação evidencia também que os alunos com diferentes níveis de competência social se distinguem significativamente em comportamentos relevantes para a realização de tarefas em sala de aula, tais como a exatidão das respostas que dão, o comportamento de escuta, a capacidade de levar a cabo uma tarefa, de seguir instruções e o tempo na tarefa (Gresham \& Reschly, 1986). Contudo não há evidência suficiente que apóie a idéia de uma relação de causalidade entre as dificuldades de aprendizagem e os déficits competência social, e não foram também encontrados marcadores de competências sociais específicos para as crianças com e sem dificuldades de aprendizagem.

Apesar da associação entre os níveis de desempenho acadêmico e social, o debate continua relativamente à explicação destas relações. Hipóteses rivais têm sido defendidas. Alguns autores consideram que as dificuldades sociais dos alunos e as dificuldades de aprendizagem têm ambas origem num mesmo e único déficit cognitivo geral. Ou seja, as dificuldades de aprendizagem destes alunos estender-se-iam quer à área acadêmica, quer à sua capacidade de aprendizagem social, a qual seria então vista como uma dificuldade específica de aprendizagem (ICLD, 1987). Numa outra perspectiva sugere-se que os déficit na competência social podem ser efeitos secundários das dificuldades acadêmicas. $\mathrm{O}$ aluno com dificuldades acadêmicas significativas ficaria, em consequiência, numa posição social desfavorecida, à qual ele próprio e os outros (pais, professores, colegas) reagem negativamente, criando assim condições propícias para a perpetuação destes efeitos cíclicos debilitantes. Ou seja, segundo esta concepção, as dificuldades acadêmicas e as dificuldades sociais não decorrem de um mesmo déficit de base. Antes, os déficit na competência social seriam consequiência dos efeitos sociais de um baixo nível de desempenho acadêmico. Em suma, os estudos mostram elevadas correlações sem que esteja esclarecida a relação funcional entre as competências acadêmicas e sociais.

Seja como for, a competência na área acadêmica é uma tarefa fundamental do desenvolvimento, sobretudo para os indivíduos em idade escolar, constituindo um importante indicador do funcionamento social adaptado e da adaptação comportamental mais vasta. $\mathrm{E}$, para o esclarecimento da natureza e do percurso de desenvolvimento dos déficit de competência social, nomeadamente nos alunos com dificul- dades de aprendizagem e de baixo rendimento escolar, é essencial dispor de uma boa tecnologia de avaliação.

\section{As Escalas para Professores: Objetivos do Estudo}

Este estudo procurou precisamente examinar as dimensões da competência social em crianças portuguesas usando as escalas da forma para professores (nível pré- escolar, que compreende os graus de escolaridade que se situam entre o último ano da pré-escola e o $6^{\circ}$ ano) do SSRS. O interesse por este instrumento resulta da importância que a competência social assume em contexto escolar e da necessidade de colocar ao dispor da investigação e da intervenção um instrumento de avaliação adaptado à população infantil portuguesa.

Os professores são uma fonte particularmente útil de informação acerca do comportamento do aluno na sala de aula e da sua competência acadêmica, porque as suas avaliações se baseiam em grandes amostras de comportamento observado ao longo de extensos períodos de tempo. A forma para professores do SSRS avalia essencialmente as habilidades sociais, mas também problemas de comportamento (que possam interferir com a aquisição ou desempenho das habilidades sociais) e a competência acadêmica. A avaliação do funcionamento nestas três áreas poderá indicar possíveis causas dos problemas sociais de alguns alunos, o que por sua vez facilitará o planejamento de intervenções de pequeno espectro e de elevada precisão.

A construção destas escalas baseou-se na investigação sobre o comportamento e desenvolvimento social das crianças. Mais especificamente a elaboração dos diversos itens alicerçou-se em dados de investigação empírica oriundos de vários domínios: psicologia do desenvolvimento, psicologia clínica, psicologia da educação e educação especial.

A escala de habilidades sociais, avalia comportamentos nucleares de três componentes: a cooperação (dez itens) que inclui comportamentos de ajuda, partilha e cumprimento de normas (exemplo: "Termina as tarefas de sala de aula a tempo"); a asserção (dez itens) que avalia a freqüência com que o aluno toma iniciativas como pedir informações ou apresentar-se e as suas respostas e reações aos outros (exemplo: "Faz amigos facilmente"); e o autocontrole (dez itens) que avalia comportamentos como reagir adequadamente em situações conflituosas, assumir compromissos e esperar pela sua vez (exemplo: "Reage de forma adequada quando empurrado ou agredido por outras crianças"). O conflito e a coordenação, contemplados nestas sub-escalas, têm sido considerados fundamentais para a compreensão dos processos sociais (Killen, 1989). De fato, as situações de conflito permitem compreender como os indivíduos negociam e interpretam os acontecimentos e comportamentos, nos seus diversos contextos. Por sua vez, a coordenação social é um processo que revela o modo como o indivíduo interage com os outros e como lhes responde.

A escala de problemas de comportamento avalia três tipos de problemas inadequados: problemas externalizados 
(seis itens) que avaliam a agressão física ou verbal, contestação, baixo controlo do comportamento próprio (exemplo: "Ameaça e goza os colegas"); problemas internalizados (seis itens) representados por indicadores de ansiedade, tristeza, solidão, e baixa auto-estima (exemplo: "Isola-se"); e a hiperatividade (seis itens) que inclui a agitação, excitação e reações impulsivas (exemplo: "Perturba as atividades").

Nestas duas escalas, baseando-se na freqüência do comportamento, o professor registra se este ocorre "nunca", "às vezes", ou "muitas vezes".

Quanto à competência acadêmica, a escala é constituída por comportamentos críticos tais como o desempenho na leitura e na matemática, a motivação, o funcionamento cognitivo global e o suporte parental. Os nove itens que a compõem são cotados numa escala de cinco pontos em que " 1 " corresponde ao nível mais baixo e " 5 " ao mais elevado.

Mais especificamente o presente estudo teve os seguintes objetivos: (a) explorar a configuração estrutural dos construtos do SSRS verificando se as respostas dos professores acerca das crianças portuguesas refletem o padrão pressuposto das dimensões da competência social, (b) explorar a importância relativa das diferentes dimensões das habilidades sociais e dos problemas de comportamento no resultado total das respectivas escalas, nas crianças portuguesas, (c) examinar as relações entre as três escalas componentes da competência social avaliadas por este instrumento.

\section{Método}

\section{Sujeitos}

Os participantes foram 342 alunos, 169 dos quais $(49,42 \%)$ do $3^{\circ}$ ano de escolaridade e $173(50,58 \%)$ do $6^{\circ}$ ano, de quatro escolas do $1^{\circ}$ ciclo e de duas escolas do $2^{\circ}$ ciclo do ensino básico. Os alunos distribuíam-se eqüitativamente quanto ao sexo: no $3^{\circ}$ ano $47,34 \%$, e no $6^{\circ}$ ano $47,98 \%$, eram do sexo masculino, e os restantes do sexo feminino. As idades dos alunos do $3^{\circ}$ ano variavam entre 8 e 11 anos, com uma média de 8 anos e 4 meses e as dos alunos do $6^{\circ}$ ano variavam entre 11 e 15 anos, com uma média de 11 anos e 8 meses. Estes alunos pertenciam a 17 turmas ( 9 turmas do $3^{\circ}$ ano e 8 turmas do $6^{\circ}$ ano de escolaridade). No estudo colaboraram também 17 professores, um de cada uma destas turmas.

\section{Instrumentos}

\section{A versão Portuguesa}

Os itens das escalas foram traduzidos e testados através de entrevistas e reflexão falada com professores acerca das crianças portuguesas para verificar a adequação da compreensão do que se pretendia avaliar. A escala de competência acadêmica apresenta formulações diferentes do item relativo ao desempenho na disciplina de Português: no $3^{\circ}$ ano o item inquire sobre a "leitura" e no $6^{\circ}$ ano sobre a "Língua Portuguesa". Esta escala comporta, na versão portuguesa apenas seis itens, tendo sido excluídos o suporte parental, o comportamento global e a motivação global.

\section{Procedimentos}

Os professores preencheram individualmente as três escalas para cada um dos alunos. No $3^{\circ}$ ano, cada professor preencheu as escalas para todos os alunos da sua turma. No $6^{\circ}$ ano, esta tarefa coube ao diretor de turma, dado ser este o professor que tem um melhor conhecimento da turma e dos seus alunos.

\section{Análise dos Dados}

A análise de dados procurou examinar se as dimensões das três escalas de competência social (habilidades sociais, problemas de comportamento e competência acadêmica) estipuladas pelo modelo dos autores emergiam das respostas dos professores sobre os seus alunos e a importância que cada dimensão assume nos componentes mais vastos da competência social. Para tal, analisaram-se as características das escalas ao nível dos itens (estrutura fatorial) e ao nível da escala (consistências internas e médias das escalas).

Os itens de cada escala, classificados de 1 a 3 (em que 1 significa que o comportamento nunca ocorre, 2 significa que o comportamento ocorre algumas vezes e 3 que o comportamento ocorre muitas vezes), foram submetidos a uma análise fatorial dos componentes principais com rotação ortogonal $(\text { varimax })^{2}$.

Foram realizadas três análises fatoriais independentes, uma para cada escala (habilidades sociais, problemas de comportamento e competência acadêmica).

As intercorrelações entre fatores foram calculadas através do coeficiente de correlação de Pearson.

\section{Resultados}

\section{Características dos Itens}

Consistentemente com a concepção que presidiu ao SSRS a análise de cada uma das duas primeiras escalas deveria resultar em três fatores, e a da última escala em um fator único. Os fatores da escala das habilidades sociais deveriam ser três, definidos por itens de cooperação, asserção e autocontrole, respectivamente. Os fatores da escala dos problemas de comportamento deveriam ser três, definidos por itens de problemas externalizados, internalizados e de hiperatividade.

Como se pode observar nas tabelas 1 a 3 , de forma geral os resultados estão de acordo com o esperado.

2 Com exceção da escala de competência académica, para a qual se espera apenas um factor. Nesta escala os itens são classificados de 1 a 5 , em que 1 significa um desempenho muito baixo, 2 um desempenho baixo, 3 um desempenho médio, 4 um desempenho elevado e 5 um desempenho muito elevado. 


\section{Competência Social}

Na escala de habilidade sociais, apenas um item (cooperação 26) não apresenta uma saturação superior a 0,30 , no fator a que deveria pertencer. Este item foi por isso excluído da escala. Dois itens (asserção 19 e autocontrole 22) apresentam saturações em fatores diferentes dos da escala original: o primeiro passa a integrar a escala de autocontrole e o segundo a escala de asserção.

$\mathrm{Na}$ escala problemas de comportamento, apenas um item (hiperatividade 47) apresenta um resultado que não confirma a sua pertença a esta sub-escala; o item inclui-se de forma muito satisfatória nos problemas externalizados.

$\mathrm{Na}$ escala de competência acadêmica, os resultados confirmam integralmente a estrutura pressuposta.

Em suma, no geral os resultados constituem uma contrapartida empírica da pluralidade teórica de dimensões implicadas na competência social.

As correlações entre fatores (tabela 4) indicam relações fortes entre as dimensões cooperação, autocontrole e asserção da escala de Habilidades Sociais.

Quanto à escala de Problemas de Comportamento, as relações entre problemas externalizados e internalizados e

Tabela 1. Itens e estrutura fatorial da escala de habilidades sociais.

\begin{tabular}{|c|c|c|c|}
\hline \multicolumn{4}{|c|}{ Estrutura Fatorial } \\
\hline Itens & $\begin{array}{c}\text { Auto- } \\
\text { controle }\end{array}$ & $\begin{array}{c}\text { Coope- } \\
\text { ração }\end{array}$ & $\begin{array}{c}\text { Asser- } \\
\text { ção }\end{array}$ \\
\hline 12. Controla temperamento (c/ adultos) & 0,75 & & \\
\hline 11. Adequado(a) sob desafio & 0,75 & & \\
\hline 25. Adequado(a) sob provocação & 0,74 & & \\
\hline 13. Aceita críticas & 0,72 & & \\
\hline 5. Adequado(a) sob pressão & 0,71 & & \\
\hline 18. Aceita idéias & 0,71 & & \\
\hline 1. Controla temperamento (c/ colegas) & 0,70 & & \\
\hline 4. Negocia & 0,60 & & \\
\hline 30. Dá-se com pessoas diferentes & 0,47 & & \\
\hline 19. Elogia colegas & 0,45 & & \\
\hline 27. Organizado(a) & & 0,75 & \\
\hline 16. Trabalhos cuidados & & 0,74 & \\
\hline 9. Termina tarefas em tempo & & 0,71 & \\
\hline 21. Arrumado(a) & & 0,70 & \\
\hline 28. Obedece ao professor & & 0,70 & \\
\hline 15. Cumpre normas & & 0,68 & \\
\hline 20. Segue orientações & & 0,64 & \\
\hline 8. Ocupação adequada dos tempos livres & & 0,63 & \\
\hline 29. Segue facilmente transições & & 0,61 & \\
\hline 26. Ignora distrações & & 0,11 & \\
\hline 7. Convida colegas a participar & & & 0,78 \\
\hline 2. Inicia novos contactos & & & 0,78 \\
\hline 14. Enceta conversas com colegas & & & 0,68 \\
\hline 3. Questiona regras adequadamente & & & 0,66 \\
\hline 24. Integra-se em grupos & & & 0,65 \\
\hline 10. Faz amigos facilmente & & & 0,64 \\
\hline 23. Oferece ajuda & & & 0,62 \\
\hline 22. Inicia colaboração & & & 0,59 \\
\hline 6. Auto-elogio adequado & & & 0,57 \\
\hline 17. Analisa injustiças com professor & & & 0,31 \\
\hline
\end{tabular}

Tabela 2. Itens e estrutura fatorial da escala de problemas de comportamento.

\begin{tabular}{lccc}
\hline \multicolumn{4}{c}{ Estrutura Fatorial } \\
\hline Itens & $\begin{array}{c}\text { P. } \\
\text { Externali- } \\
\text { zadas }\end{array}$ & $\begin{array}{c}\text { P. } \\
\text { Internali- } \\
\text { zadas }\end{array}$ & $\begin{array}{c}\text { Hiperati- } \\
\text { vidade }\end{array}$ \\
\hline 44. Irrita-se & 0,80 & & \\
42. Protesta com adultos & 0,79 & & \\
33. Ameaça e goza & 0,79 & & \\
31. Luta & 0,78 & & \\
43. Zanga-se & 0,76 & & \\
47. Impulsivo (a) & 0,75 & & \\
41. Discute & 0,74 & & \\
34. Solitário (a) & & 0,83 & \\
46. Triste & & 0,82 & \\
45. Isolado (a) & & 0,77 & \\
32. Baixa auto-estima & & 0,72 & \\
39. Envergonhado (a) & & 0,65 & \\
38. Ansioso (a) em grupo & & & 0,51 \\
35. Distrai-se & & & 0,69 \\
40. Desatento (a) às conversas & & & 0,66 \\
36. Interrompe & & & 0,66 \\
37. Perturbador & & & \\
48. Inquieto(a) & & & \\
\hline
\end{tabular}

Tabela 3. Itens e estrutura fatorial da escala de competência acadêmica.

\begin{tabular}{lc}
\hline Item & $\begin{array}{c}\text { Estrutura } \\
\text { fatorial }\end{array}$ \\
\hline 49. Desempenho acadêmico global (critério relativo) & 0,94 \\
54. Funcionamento intelectual (critério relativo) & 0,92 \\
53. Aptidão Matemática (critério absoluto) & 0,91 \\
51. Matemática (critério relativo) & 0,90 \\
52. Aptidão Leitura/Língua Portuguesa (critério & 0,89 \\
$\quad$ absoluto) & 0,88 \\
50. Leitura/Língua Portuguesa (critério relativo) & \\
\hline
\end{tabular}

Notas. Critério relativo: Como é que o aluno se situa em relação aos colegas; Critério absoluto: Relativamente ao que seria de esperar para o $3^{\circ} 6^{\circ}$ ano de escolaridade. No $3^{\circ}$ ano pergunta-se sobre a "Leitura" e no $6^{\circ}$ sobre a "Língua Portuguesa".

entre problemas internalizados e hiperatividade são baixas, para o $3^{\circ}$ e $6^{\circ}$ anos de escolaridade. Os problemas externalizados apresentam relações fortes com a hiperatividade quer no $3^{\circ}$ quer no $6^{\circ}$ ano.

Evidenciaram-se correlações genericamente significativas entre os componentes das Habilidades Sociais e os componentes dos Problemas de Comportamento. Como seria de esperar estas correlações são negativas. Isto é, quanto maiores os Problemas de Comportamento, mais baixas as Habilidades Sociais, e vice-versa.

Como se pode observar na tabela 4 , as relações entre escalas e sub-escalas são mais fortes para o $6^{\circ}$ do que para $o$ $3^{\circ}$ ano de escolaridade. Estes resultados sugerem que ao longo do desenvolvimento o nível de desempenho num aspecto específico terá repercussões cada vez mais fortes sobre os outros componentes da competência social. 
Tabela 4. Correlações entre as dimensões das escalas de habilidades sociais e de problemas de comportamento.

\begin{tabular}{llllll}
\hline \multirow{2}{*}{ Dimensões } & \multicolumn{2}{c}{ Habilidades Sociais } & \multicolumn{1}{c}{$\begin{array}{c}\text { Problemas de } \\
\text { Comportamento }\end{array}$} \\
\cline { 2 - 6 } & Coop. & Assert. Auto-C. Extern. Intern. Hiperact \\
\hline $3^{\text {o ano }}$ & & & & & \\
Assertividdade & $0,43^{* *}$ & & & & \\
Auto-controlo & $0,59^{* *}$ & $0,35^{* *}$ & & & \\
P. Externaliz. & $-0,50^{* *}$ & 0,01 & $-0,74^{* *}$ & & \\
P. Internaliz. & $-0,43^{* *}$ & $-0,55^{* *}$ & $-0,29^{* *}$ & $0,18^{*}$ & \\
Hiperactivid. & $-0,63^{* *}$ & $-0,13$ & $-0,64^{* *}$ & $0,69^{* *}$ & $0,24^{* *}$ \\
\hline $6^{\circ}$ ano & & & & & \\
Assertividade & $0,59^{* *}$ & & & & \\
Auto-controlo & $0,70^{* *}$ & $0,46^{* *}$ & & & \\
P. Externaliz. & $-0,66^{* *}$ & $-0,26^{* *}$ & $-0,78^{* *}$ & & \\
P. Internaliz. & $-0,31^{* *}$ & $-0,45^{* *}$ & $-0,08$ & $0,24^{* *}$ & \\
Hiperactivid. & $-0,69^{* *}$ & $-0,29^{* *}$ & $-0,65^{* *}$ & $0,81^{* *}$ & $0,25^{* *}$ \\
\hline$* * p<0,01 ; * p<0,05$. & & & & \\
** $p<0$. & & &
\end{tabular}

Os resultados reforçam a pertinência de uma concepção multidimensional da competência social. O estudo empírico deste instrumento mostrou que apesar de as três dimensões de cada escala serem distintas, elas se relacionam entre si, contribuindo para uma definição simultaneamente compreensiva e diferenciada da competência social. Este tipo de instrumento, baseado neste conceito, permite a análise mais detalhada e precisa da competência social, como, por exemplo, estudos sobre os efeitos diferenciais de aspectos específicos da competência social, estudos sobre as trajetórias de desenvolvimento de cada uma das suas dimensões e ainda sobre as relações entre os vários aspectos da competência social em diferentes idades e tipos de população.

Quanto às escalas totais, as habilidades sociais apresentam uma relação negativa, estatisticamente significativa $(\mathrm{p}<0,01)$ com os problemas de comportamento, quer no $3^{\circ}$ ano $(-0,67)$, quer no $6^{\circ}$ ano $(-0,71)$.

Para avaliar a relação entre competência acadêmica e as outras duas escalas, definiram-se dois grupos de alunos, um com realização acadêmica baixa e o outro com realização acadêmica média/elevada, usando como critério os percentis (o percentil 10 e o percentil 90, com base nos quais se definiram primeiro 3 grupos - baixo, médio e elevado). Seguidamente juntaram-se os dois últimos num só, uma vez que se pretendia comparar os alunos com realização acadêmica baixa, com os alunos com realização acadêmica média/elevada, à semelhança dos outros estudos nesta área. De fato, não há razões conceptuais nem empíricas para pensar que os alunos com realização média e elevada se distingam significativamente quanto à sua competência social. $\mathrm{O}$ grupo de realização média/elevada foi então contrastado com o grupo de realização baixa. Averiguou-se a existência de diferenças nas escalas de habilidades sociais e de problemas de comportamento nos dois grupos de realização acadêmica, em cada ano de escolaridade, através da análise de variância (tabelas 5 e 6). Os alunos com baixa realização acadêmica apresentaram significativamente menos habilida-
Tabela 5. Diferenças entre os grupos de realização acadêmica baixa (G1) e média/elevada (G2), nas Escalas de Habilidades Sociais e de Problemas de Comportamento, nos Alunos do $3^{\circ}$ Ano.

\begin{tabular}{|c|c|c|c|c|c|}
\hline \multirow{2}{*}{ Escalas } & \multicolumn{2}{|c|}{ G1 } & \multicolumn{2}{|c|}{ G2 } & \multirow{2}{*}{ F $(1,166)$} \\
\hline & M & (DP) & M & (DP) & \\
\hline Habilidades sociais & 51,27 & 11,26 & 69,82 & 16,19 & $23,43^{* *}$ \\
\hline Probls. de comportamento & 47,37 & 17,28 & 34,48 & 18,92 & $8,01 * *$ \\
\hline
\end{tabular}

$* * p<0,01$

Tabela 6. Diferenças entre os grupos de realização acadêmica baixa (G1) e média/elevada (G2), nas escalas de habilidades sociais e de problemas de comportamento, nos alunos do $6^{\circ}$ ano.

\begin{tabular}{|c|c|c|c|c|c|}
\hline \multirow{2}{*}{ Escalas } & \multicolumn{2}{|c|}{ G1 } & \multicolumn{2}{|c|}{ G2 } & \multirow{2}{*}{ F $(\mathbf{1 , 1 7 0 )}$} \\
\hline & M & (DP) & M & (DP) & \\
\hline Habilidades sociais & 45,98 & 11,89 & 64,86 & 15,72 & $24,54 * *$ \\
\hline P. de comportamento & 51,23 & 15,49 & 30,43 & 20,06 & $18,06^{* *}$ \\
\hline
\end{tabular}

des sociais e mais problemas de comportamento do que os alunos com uma realização acadêmica média/elevada.

Nestas tabelas podem observar-se diferenças estatisticamente significativas entre os dois grupos de realização, relativamente às duas componentes da competência social ${ }^{3}$.

\section{Características das Escalas}

A consistência interna (alpha de Cronbach) foi avaliada para cada uma das escalas. Relativamente à escala de habilidades sociais os valores para a escala total e sub-escalas de cooperação, asserção e autocontrole foram de 0,93, 0,91, 0,86 e 0,90 , respectivamente. Relativamente à escala de problemas de comportamento os valores para a escala total e sub-escalas de problemas externalizados, internalizados e hiperatividade foram de $0,91,0,92,0,83$ e 0,87 , respectivamente. No que se refere à escala de realização acadêmica o valor foi de 0,96 .

As médias das escalas e sub-escalas ${ }^{4}$ foram mais elevadas para as habilidades sociais, apresentando valores francamente positivos.

Note-se que os resultados individuais nesta escala não são uniformemente elevados, registrando-se valores mínimos de 0 nas sub-escalas de cooperação e de asserção. Como pode observar-se nesta tabela, esta escala apresenta uma ligeira assimetria positiva e é ligeiramente platicúrtica ${ }^{5}$ no $3^{\circ}$ ano de escolaridade, indicando que a média deste grupo se aproxima um pouco mais dos valores mínimos do que dos

3 Os valores aqui apresentados fazem parte de uma análise multivariada que inclui mais variáveis.

4 Os itens foram cotados de forma a que quanto maior a sua pontuação, mais elevado o valor no aspecto em avaliação. Uma vez que as subescalas eram constituídas por diferente número de itens, com o intuito de tornar directamente comparáveis as escalas e sub-escalas entre si, optou-se por recorrer a uma transformação linear das mesmas, com base na seguinte fórmula: $100 *$ [(cotação-min)/(max-min) $]$. Assim, as cotações são expressas numa escala de 0 a 100, transformação que 


\section{Competência Social}

máximos; ou seja, alguns destes alunos têm níveis especialmente elevados de habilidades sociais. A ligeira assimetria positiva da escala de Problemas de Comportamento para o $3^{\circ}$ ano sugere igualmente que de entre estes alunos, alguns demonstram níveis particularmente elevados de problemas deste tipo. Todas as outras escalas apresentam uma distribuição simétrica e mesocúrtica, quer para o $3^{\circ}$, quer para o $6^{\circ}$ ano de escolaridade. Os problemas de comportamento, embora com uma média mais baixa, não deixam de ser assinalados pelos professores. De entre estes, destaca-se a hiperatividade, (sobretudo no $3^{\circ}$ ano). Considerando a importância que este tipo de comportamentos assume em contexto de sala de aula, estes valores indicam a presença de dificuldades no ambiente de ensino/aprendizagem. A competência acadêmica apresenta valores médios moderados, sugerindo que os professores fazem uma apreciação satisfatória do desempenho dos seus alunos. Contudo os valores nesta escala, seja para o $3^{\circ}$ ano, quanto para o $6^{\circ}$ ano, variam entre um mínimo de 12,5 e um máximo de 100 , o que mostra que há uma importante variabilidade inter-individual quanto a esta competência.

\section{Discussão}

Os resultados apóiam uma concepção multidimensional da competência social e são semelhantes aos apresentados em estudos prévios (Gresham \& Elliott, 1990). De forma geral, os resultados suportam a idéia de que há uma variedade de fatores implicados no funcionamento social. Mais especificamente, a cooperação, asserção e autocontrole constituem dimensões diferentes mas fundamentais das habilidades sociais. Do mesmo modo, os problemas externalizados, internalizados e a hiperatividade constituem componentes distintos, mas indispensáveis dos problemas de comportamento. Além disso, as habilidades sociais, os problemas de comportamento e a competência acadêmica apresentam relações significativas entre si, parecendo oferecer contribuições distintas e complementares ao funcionamento social adaptado e desadaptado. Ou seja, as sub-escalas são diferençáveis (conforme mostrou a análise fatorial) e simultaneamente relacionadas entre si e as três escalas apresentam também inter-relações, as quais têm implicações importantes para o esclarecimento da complexidade do fenômeno da competência social. Em resumo, a identificação e distinção conceptual de componentes da competência social são empiricamente replicáveis.

Quanto à importância relativa das componentes das escalas, é na escala de problemas de comportamento que se notam diferenças. De entre os três sub-tipos de problemas de comportamento destaca-se a hiperatividade; estes resultados indicam pois um alvo preferencial de prevenção e aten-

não acarretou qualquer tipo de alteração em termos dos resultados nos diversos testes estatísticos.

5 O valor da assimetria é ligeiramente mais elevado do que o dobro do erro da assimetria e a razão curtose/erro da curtose é $=-2,768(>-2)$. ção em contexto de sala de aula. Não se verificaram discrepâncias dignas de registro na escala de habilidades sociais. Assim, o nível de desempenho no que se refere à cooperação, asserção e autocontrole é semelhante, pelo que estas três variáveis deverão ser abordadas de uma forma integrada.

Os resultados sugeriram também a existência de uma dependência progressivamente mais estreita entre as dimensões específicas da competência social, ao longo do desenvolvimento, sublinhando o interesse da prevenção e das intervenções em idades precoces.

A adaptação dos itens à realidade das escolas portuguesas revelou que estes são socialmente válidos, como é sugerido pelo fato de os professores terem utilizado todos os níveis de cotação previstos na escala.

As características do instrumento garantem a qualidade da informação recolhida, no que se refere à validade (validade de construto, avaliada através da validade fatorial). As escalas são compostas por um número reduzido de itens, aumentando a facilidade e eficiência na sua aplicação. Apesar do pequeno número de itens, observaram-se valores altamente satisfatórios de consistência interna. $\mathrm{O}$ instrumento constitui, portanto, uma boa ferramenta para o progressivo esclarecimento da temática.

Concluindo, pode dizer-se que as escalas de avaliação da competência social de alunos (forma para professores) são um instrumento válido, confiável e útil para uma caracterização detalhada e compreensiva da competência social em contexto escolar e para o planejamento e monitoração de intervenções destinadas a aperfeiçoar virtualidades e corrigir déficits nesta área.

\section{Referências}

Achenbach, T. (1982). Developmental psychopathology. (2nd Org.). New York: Wiley.

Bryan, T. (1982). Social skills of learning disabled children and youth: An overview. Learning Disability Quarterly, 5, 332-343.

Clark, L., Gresham, F.M., \& Elliott, S.N. (1985). Development and validation of a social skills assessment measure: The TROSSC. Journal of Psychoeducational Assessment, 4, 347-356.

Coie, J.D., \& Dodge, K.A. (1983). Continuity of children's social status: A five-year longitudinal study. Merrill-Palmer Quarterly, 29, 261-282.

Coie, J.D., Dodge, K.A., \& Copotelli, H. (1982). Dimensions and types of social status: A cross-age perspective. Developmental Psychology, 18, 557-570.

Cowen, E.L., Pederson, A., Babigian, H., Izzo, L.D., \& Trost, M.D. (1972). Long term follow-up of early detected vulnerable children. Journal of Consulting and Clinical Psychology, 41, 438446.

Deshler, D., Schumaker, J., Warner, M., Alley, G., \& Clark, F. (1980). An epidemiological study of learning disabled adolescents in secondary schools: Social status, peer relationships, activities in and out of school, and time uses (9). Lawrence: The University of Kansas Institute for Research in Learning Disabilities. 


\section{S. Lemos e H. I. Meneses}

Dodge, K.A. (1985). Facets of social interaction and the assessment of social competence in children. Em B. H. Scheider, K. H. Rubin, \& J.E. Ledingham (Orgs.), Children's peer relations: Issues in assessment and intervention (pp. 9-22). New York: Springer Verlag.

Edelbrock, C. (1983). Problems and issues in using rating scales to assess child personality and psychopathology. School Psychology Review, 12, 293-299.

Elliott, S.N., Gresham, F.M., Freeman, T., \& McCloskey, G. (1988). Teacher and observer ratings of children's social skills: Validation of the Social Skills Rating Scales. Journal of Psychoeducational Assessment, 6, 152-161.

Garmezey, N., Masten, A.S., \& Tellegen, A. (1984). The study of stress and competence in a building block for developmental psychopathology. Child Development, 55, 97-11.

Gresham, F.M. (1981a). Social skills training with handicapped children: A review. Review of Educational Research, 51, 139176.

Gresham, F.M. (1981b). Validity of social skills measures for assessing social competence in low.status children: A multivariate investigation. Developmental Psychology, 17, 390-398.

Gresham, F.M., \& Elliott, S.N. (1984). Assessment and classification of children's social skills: A review of methods and issues. School Psychology Review, 13, 292-301.

Gresham, F.M., \& Elliott, S.N. (1990). Social skills rating system: Manual. Circle Pines, MN: American Guidance Service.

Gresham, F.M., \& Reschly, D.J. (1986). Social skills and low peer acceptance of mainstreamed learning disabled children. Learning Disability Quarterly, 9, 23-32.

Gresham, F. M., Elliott, S. N., \& Black, F.L. (1987a). Factor structure replication and bias investigation of the Teacher Rating of Social Skills. Journal of School Psychology, 25, 85-92.

Gresham, F.M., Elliott, S.N., \& Black, F.L. (1987b). Teacher-rated social skills of mainstreamed mildly handicapped and nonhandicapped children. School Psychology Review, 16, 7888 .

Gresham, F.M., \& Reschly, D.J. (1987). Sociometric differences between mildly handicapped and nonhandicapped black and white students. Journal of Educational Psychology, 79, 195197.

Haager, D., \& Vaughn, S. (1995). Parent, teacher, peer, and selfreports of the social competence of students with learning disabilities. Journal of Learning Disabilities, 28(4), 205-215.

Hazel, J.S., \& Schumaker, J.B. (1988). Social skills and learning disabilities: Curent issues and recommendations for future research. Em J.F. Kavanagh \& T.J. Truss, Jr. (Orgs.), Learning disabilities: Proceedings of the national conference (pp. 293344). Parkton, MD: York Press.

I.C.L.D. (1987). Learning disabilities: A report to the U. S. Congress. Bethesda, MD: National Institutes of Health.

Kaufman, M., Agard, J., \& Semmel, M. (1985). Mainstreaming: Learners and their environment. Cambridge, MA: Brookline Books.

Killen, N. (1989). Context, conflict, and coordination in social development. Em L.T. Winegar (Org.), Social interaction and the development of children's understanding (pp. 119-146). Norwood, NJ: Ablex.

Markus, H., \& Wurf, E. (1987). The dynamic self-concept: A social psychological perspective. Annual Review of Psychology, 33, 299-337.

Moreno, J. (1934). Who shall survive? A new approach to the problem of human interrelations. Washington, DC: Nervous and Mental Disease Publishing Co.

Openheimer, L. (1987). Cognotive and social variables in the plan of action. Em S.F. Friedman, E.K. Scholnick, \& R.R. Cocking (Orgs.), Blueprints for thinking: The role of planning in cognitive development (pp. 346-392). New York: Cambridge University Press.

Openheimer, L. (1989). The nature of social action: Social competence versus social conformism. Em B.H. Scneider, G. Attili, J. Nadel, \& R.P. Weissberg (Orgs.), Social competence in developmental perspective (pp. 41-68). New York: Kluer Academic.

Parker, J.G., \& Asher, S.R. (1987). Peer relations and later personal adjustment: Are low-accepted children at risk? Psychological Bulletin, 102, 357-389.

Quay, H. (1983). A dimensional approach to behavior disorder: The Revised Behavior Problem Checlist. School Psychology Review, 12, 244-249.

Reschley, D.J., \& Gresham, F.M. (1988). Adaptive behavior and the mildly handicapp. Em T. Kratochwill (Org.), Advances in school psychology (pp. 249-282). Hillsdale, NJ: Erlbaum.

Stephens, T. (1978). Social skills in the classrom. Columbus, OH: Cedars Press.

Stephens, T. (1981). Technical information: Social Behavior Assessment. Columbus, OH: Cedars Press.

Vaugh, S. \& Hogan, A. (1990). Social Competence and learning disabilities: A prospective study. Em A.L. Swanson, \& B.K. Keogh (Orgs.), Learning disabilities: Theoretical and research issues (pp. 175-191). Hullsdale, NJ: Erlbaum.

Walker, H.M., \& McConnell, S. (1988). Walker-McConnell Scale of Social Competence and School Adjustment. Austin, TX: PROORG. 